\title{
A micro-mechanical evaluation of the effects of die hardener on die stone
}

\author{
Li-Hong He, Ludwig Jansen van Vuuren, Nina Planitz and Michael V. Swain \\ Sir John Walsh Institute, School of Dentistry, University of Otago, PO Box 647, Dunedin 9054, New Zealand \\ Corresponding author, Li-Hong He; E-mail: lihong.he@otago.ac.nz
}

\begin{abstract}
The purpose of this study was to investigate the properties of a die hardener penetrated layer and evaluate its protective effects on the surface of die stone. A commercial die hardener (PDQ die hardener, Whipmix corp., USA) was tested on a die stone (GC Fujirock ${ }^{\circledR}$ EP die stone, GC Europe, Belgium) and a dental plaster (Dental Stone, United States Gypsum Company, USA). Nanoindentation and micro-scratch tests were performed on both coated and uncoated specimens. The scratch damage was observed by SEM and the penetration depth of die hardener was detected by the affiliated EDX. Upon drying, the die hardener penetrated into the die stone to a depth of 3-5 $\mu \mathrm{m}$, and deposited a thin film on the surface of die stone. Although the die hardener penetrated layer did not show improved mechanical properties, the die hardener film on the surface did protect the specimens from abrasion damage.
\end{abstract}

Keywords: Die hardener, Nanoindentation, Micro-scratching

\section{INTRODUCTION}

Gypsum products are important materials in dentistry for the fabrication of indirect dental prostheses. To meet the dental laboratory requirements, gypsum casts and dies must be: accurate, dimensionally stable over time, hard enough to withstand the fabrication process, and resistant to the inadvertent abrasions caused during fabrication. Among the above mentioned characteristics, the abrasion resistance is one of the most important characters because most of the dental laboratory fabrication procedures are performed on gypsum casts and any material loss occurring at critical areas of the die cast (e.g. margins and interproximal contacts) may result in an unacceptable final prosthesis.

To increase abrasion resistance of cast dies, several methods have been documented. One is the use of epoxy resin die material ${ }^{1)}$. However, dimensional accuracy and high cost restrict its prevalence. Another one is the substitution of colloidal silica or soluble resin solution for water in gypsum die materials ${ }^{2}$. Unfortunately, this method may result in unpredictable setting expansion. A third approach is to coat the gypsum casts with a resin film. This became a popular technique because of the ease of application and low cost. Different materials, such as cyanoacrylate, die sealants, epoxy, acrylic styrene and resins, have been utilized as die hardeners ${ }^{3-7}$. However, the reports are contradictory regarding the efficacy of die hardener to protect the gypsum surface. Some studies stated that surface treatment improved the anti-abrasion properties of gypsum ${ }^{3-5)}$, while others concluded that the application of a surface hardener had no protective effects and even decreased the surface hardness ${ }^{6}$. Another study found that the effect of surface hardener on abrasion resistance was based on the type of gypsum used and the type of surface hardener applied ${ }^{7}$. The possible reasons for the conflicting results could be that the materials have experienced significant change over the last decades, as some of the tests were published in 1970's and 1980's, and the test methods involved were not standardised. Although it may not be appropriate, hardness with the definition of the resistance to permanent deformation has long been used to evaluate the resistance to abrasion. Knoop hardness tests were used in many previous studies to measure the surface hardness ${ }^{3-4,6)}$, but it may not be the best way to determine the surface hardness with die hardeners in these cases, as the indentation depth exceeds the penetration depth of the die hardener. Scratch tests were also considered to evaluate the abrasion resistance of a material ${ }^{7-10)}$. Nevertheless, most of the scratch tests on dental gypsum materials were performed with selfmade jigs and various tips, which make the comparison of results from different reports very difficult. In short, so far the available literature did not answer the questions about the mechanical properties of the die hardener penetrated layer and the mechanism of die hardener to protect the surface of from abrasion. Therefore, it was deemed necessary to test the die hardener with standardised and more accurate modern equipment.

Nanoindentation has the ability to measure the mechanical properties of small volume samples and has become one of the most popular methods to measure the mechanical properties of thin films ${ }^{11}$. A Microhardness tester has the ability to add a small load on a standard Vickers diamond indenter and the scratch tests within the die hardener penetrated layer would be more appropriate to evaluate the anti-abrasion ability of the die hardener. The aim of this study was to investigate the mechanical properties and antiabrasive behaviour of the thin die hardener penetrated layer at the surface of a die stone and a dental plaster. 


\section{MATERIALS AND METHODS}

\section{Specimen preparation}

A die stone (GC Fujirock ${ }^{\circledR}$ EP, GC Europe, Leuven, Belgium) and a dental plaster (Dental Stone, United States Gypsum Company, Chicago, IL, USA) were mixed according to the manufacturer's instructions under vacuum and cast with vibration. Twenty three bars were cast from die stone and five bars were made from dental plaster. The specimens were left to set for 2 hours in the Deguform silicone (Degudent, HanauWolfgang, Germany) mould before being ground and polished to the dimension of $30 \times 5 \times 3 \mathrm{~mm}$ with sandpaper (Struers, Copenhagen, Denmark) down to 4000 grit on a polishing machine (RotoPol-22, Struers, Copenhagen, Denmark). The specimens were then dried for 24 hours in an ambient environment.

Five prepared specimens from each material were selected randomly for additional polishing to $0.1 \mu \mathrm{m}$ with a diamond lapping film (Allied High Tech Products Inc., Rancho Dominguez, California, USA) for nanoindentation testing.

For those specimens that needed die hardener coating, PDQ die hardener liquid (Whipmix corp., Louisville, KY, USA) was brushed onto the surfaces twice and left to dry for one hour before the experiments.

\section{Nanoindentation}

Indentation experiments were performed using a nanobased indentation system (UMIS-2000, CSIRO, Sydney, NSW, Australia). Using a paralleling machine (Leitz, Wetzlar, Germany), the finished specimen was mounted on a metal base with a thin layer of wax. A strong magnet within the mounting base ensured that adequate contact was obtained with the test base in the UMIS system. The elastic modulus and hardness of the specimens were measured with a calibrated Berkovich indenter under a fixed load of $20 \mathrm{mN}$. Thirty six indentations in the form of $6 \times 6$ array were arranged on the polished surface with the interval of $50 \mu \mathrm{m}$ between two separate indents to avoid the influence of residual stresses from adjacent impressions. After the first test, the surface was coated with two layers of PDQ die hardener and left to dry for one hour. Then, a similar area on the coated surface was indented again with the same settings.

\section{Micro-scratch test}

After nanoindentation tests (24 hours later), the same specimens were used again for the scratching tests. Specimens were mounted on the stage of a microhardness tester (Shimadzu Type M, Seisakusho Ltd., Kyoto, Japan). A Vickers diamond indenter was utilized to indent the sample with a load of 15 grams and holding time of 45 seconds. After 30 seconds of loading, the sample was moved horizontally for about $200 \mu \mathrm{m}$ by rotating the micrometer on the sample stage to generate a scratch under the indentation load. At least 3 scratches were made on the surface of each sample.

\section{Flexural strength}

After drying, eighteen die stone specimens were divided into two groups arbitrarily and equally. Group 1 was coated with two applications of PDQ die hardener by brushing it onto one surface. Group 2 was left uncoated as control.

A flexural strength test was carried out with a universal test machine (Model 3369, Instron, Norwood, MA, USA) in a three-point bending manner with a lower span of $30 \mathrm{~mm}$ and cross head speed of $0.5 \mathrm{~mm} /$ min. The peak load at fracture was recorded and the flexural strength was calculated with the following equation:

$$
\sigma_{\mathrm{F}}=\frac{3 F L}{2 b h^{2}},
$$

where $F$ is peak load, $L$ is lower span (30 $\mathrm{mm}$ for current test), $b$ is the breadth of the specimen $(5 \mathrm{~mm})$ and $h$ is the height of the specimen $(3 \mathrm{~mm})$.

For the die hardener coated specimens, the coated surface was deliberately left as the tensile surface under three-point bending.

\section{Statistical analysis}

Two-tailed paired $t$-tests were performed by Microsoft Excel 2007 (Microsoft Inc., Redmond, WA, USA) software to compare data from nanoindentation and flexural strength tests.

\section{Scanning Electron Microscopy (SEM) observations}

A fractured flexural strength sample coated by die hardener was selected randomly. The fractured crosssectional surface was polished to $0.1 \mu \mathrm{m}$, using diamond film and coated with chromium for SEM observation and energy dispersive X-ray spectroscopy (EDX) analysis. A field emission SEM (JEOL 6700 FESEM, JEOL, Peabody, MA, USA) with a backscatter detector was employed for the observation. EDX analysis spectra of different regions of the sample were obtained on the same SEM using a spot collection method.

The scratched specimens were gold coated and observed by a Cambridge S360 SEM (Cambridge Instruments, Cambridge, UK) with a secondary electron detector.

\section{RESULTS}

\section{EDX detection}

The backscatter SEM (Fig. 1) image clearly illustrates a 3-5 $\mu \mathrm{m}$ penetration layer (the dark region) of the die hardener at the edge of the cross-sectional cut sample. EDX spectra on the specimen (spot a) contain oxygen, sulphur, and calcium peaks, which is consistent with gypsum material. EDX analysis on the die hardener penetrated region (spots $\mathrm{b}$ and $\mathrm{c}$ ) had an additional peak of carbon, which indicates that the functional constituents of the die hardener contain carbon .

\section{Nanoindentation}

A nanoindentation force-displacement curve (Fig. 2) 


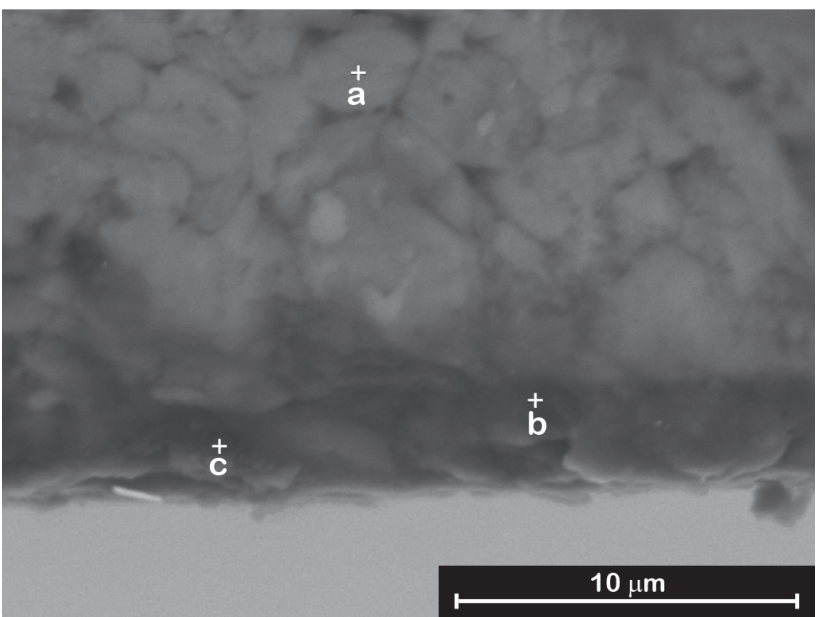

Fig. 1 Back scatter SEM image of cross-sectional cut die stone specimen for EDX analysis.

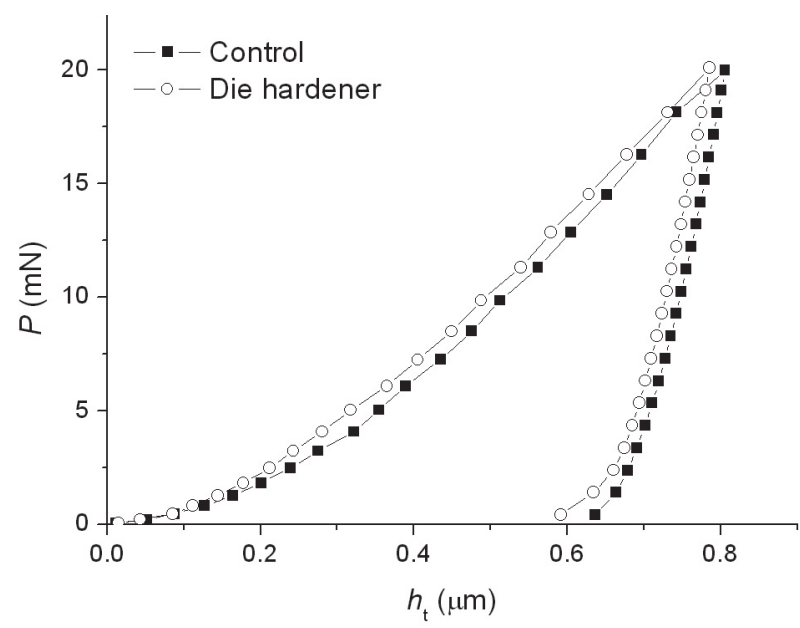

Fig. 2 Typical force-displacement curves of nanoindentation from both control and die hardener treated regions of a die stone specimen.

from the die hardener coated region of die stone specimen reveals that the indentation had a penetration depth of $-0.8 \mu \mathrm{m}$. Results from nanoindentation show that the elastic modulus and hardness of the control region were $34.71 \pm 7.52$ and $0.96 \pm 0.37 \mathrm{GPa}$, respectively. In contrast, the die hardener treated region had the elastic modulus and hardness values of $36.57 \pm 5.43$ and $1.10 \pm 0.31 \mathrm{GPa}$, respectively (Fig. 3). Student $t$-test statistical analysis indicates that there is no significant difference between the elastic moduli of the treated and control groups $(p>0.05)$. Although it seems that the die hardener increased the surface hardness slightly, there is no statistical difference between the groups $(p>0.05)$.

The nanoindentation tests on dental plaster have similar results. The elastic modulus and hardness of
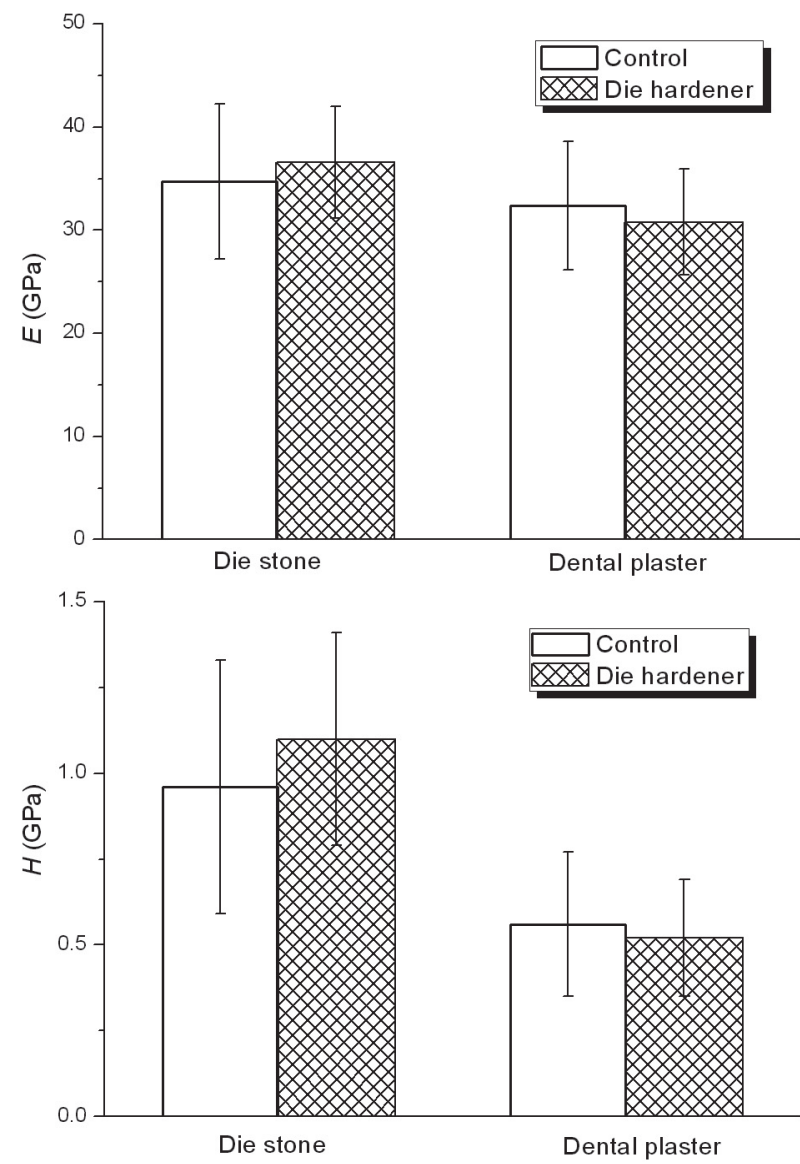

Fig. 3 Comparison of nanoindentation mechanical properties (elastic modules, $E$, and hardness, $H$ ) of control and die hardener treated surfaces of both die stone and dental plaster specimens.

the control region were $32.36 \pm 6.22$ and $0.56 \pm 0.21 \mathrm{GPa}$, respectively, while the elastic modulus and hardness of the die hardener treated region presented values of $30.79 \pm 5.13$ and $0.52 \pm 0.17 \mathrm{GPa}$, respectively (Fig. 3). No statistical difference has been recorded $(p>0.05)$.

Micro-scratch test

SEM images of typical scratches made with the Vickers indenter at a load of 15 grams $(-0.15 \mathrm{~N})$ with and without die hardener are given in Fig. 4. Figs. 4a and $4 \mathrm{c}$ illustrate extensive surface scratching damage on the uncoated surfaces of die stone and dental plaster. Under high contact pressure, the pyramid indenter considerably deformed the surfaces of the specimens and generated ploughed grooves. As a result, considerable detached debris was accumulated adjacent to the grooves. The deformation resulted in a myriad of small cracks and some associated with larger fractures that extended beyond the indenter contact region. In contrast, as can be seen in Figs. $4 \mathrm{~b}$ and $4 \mathrm{~d}$, the specimens coated by the die hardener had smoother surfaces and far less scratching damage (less debris 

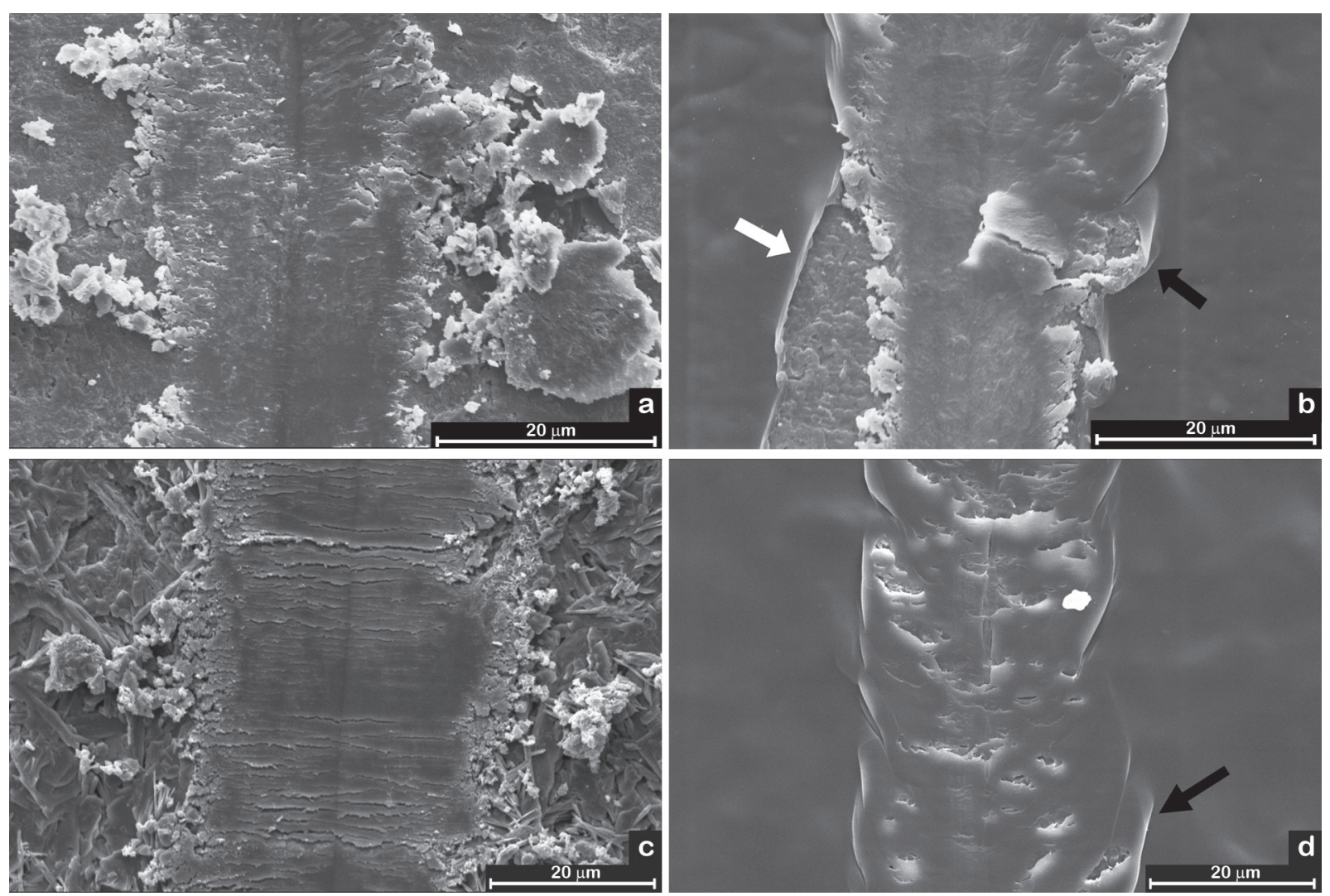

Fig. 4 SEM comparison of the surface scratches without (a) (c) and with (b) (d) die hardener coating of die stone (a) (b) and dental plaster (c) (d) specimens. The die hardener formed a film on the surface of the sample (White arrow in image b) and the plastic deformation (black arrows in image b and d) of the film protected the surface from extensive abrasion damage.

and smaller ploughed grooves). The die hardener film experienced plastic deformation on the surface of the die stone and protected the surface from excessive fracture and detachment (black arrows in Figs. $4 \mathrm{~b}$ and $4 \mathrm{~d})$. As a consequence, less surface cracks and very little debris could be observed near the ploughed grooves.

\section{Flexural strength}

Flexural strengths of the control and the coated groups were found to be $29.47 \pm 1.89$ and $29.66 \pm 1.24 \mathrm{MPa}$, respectively. Student $t$-test statistical analysis did not show any significant difference between the groups $(p>0.05)$.

\section{DISCUSSION}

Back scatter SEM and EDX observations showed that die hardener liquid infiltrated into the surface of the specimen to a depth of $3-5 \mu \mathrm{m}$ to form a die hardener penetrated layer. SEM (Figs. 4b and 4d) also illustrated that die hardener deposited a thin film on the surface of the specimens.

Unfortunately, the key component of the PDQ die hardener could not be identified. The only constituent listed in the material safety datasheet of the product is Methyl Ethyl Ketone (MEK) (with a concentration of $>50 \%$ ), which is believed to be the solvent of the product and should evaporate quickly after spreading of the product on the surface of die stone. However, EDX analysis on the die hardener penetrated layer (spots b and c in Fig. 1) revealed that the major additional element associated with the die hardener is carbon. Moreover, other literature mentioned that the active component of die hardeners may be cyanoacrylate resin, ketone-based resin, self-polymerizing oil and resin, and epoxy resin ${ }^{2-7,12)}$. Therefore, it is reasonable to speculate that the active ingredients of the PDQ die hardener are resins.

Due to the porosity of die stone and the capillary effect, the die hardener liquid infiltrated into the die stone surface at a depth of $3-5 \mu \mathrm{m}$ (Fig. 1). In this study, nanoindentation had a penetration depth of -0.8 $\mu \mathrm{m}$ on die stone and $-1.1 \mu \mathrm{m}$ on dental plaster, which was about $1 / 5$ of the total thickness of the die hardener penetrated layer. By comparing with previous microhardness tests which indented beyond the die hardener penetrated layer, current values from nanoindentation are a more accurate reflection of the mechanical properties of the die hardener penetrated layer. By 
considering the low elastic modulus and small volume fraction of the die hardener resin inside the penetrated layer, its contribution to the final elastic modulus of the composite (the mixture of gypsum crystals and die hardener resins) is minimal. As a result, it is reasonable that there was minimal difference of the elastic moduli between the coated and uncoated groups. On the hardness aspect, the current results contradict some other reports, which concluded that die hardener reduce the surface hardness ${ }^{6,12)}$. The possible reason could be that traditional micro-hardness tests used a high load and have a deeper penetration depth than current nanoindentation tests with respect to the thin die hardener penetrated layer. Therefore, the microhardness results are the reflection of a bigger volume of samples with more variance. In contrast, the current nanoindentation test has a shallower penetration depth within the die hardener penetrated layer, which reflected the effects of die hardener in a more accurate way.

After evaporation of solvents, the die hardener deposits a thin resin film on the surface of the specimen. From the micro-scratch comparison, the film does have protecting effects (Fig. 4). The possible mechanism of less scratching damage with die hardener film could be that the resin film acted as a solid lubricant and decreased the coefficient of friction between the diamond indenter and die stone. Moreover, the plastic deformation of the film (black arrows in Figs. 4b and 4d) redistributed local stresses and shielded the die stone from the destructive frictional force. This contention is supported by the noticeable reduction in the number of cracks perpendicular to the scratching direction for the die hardener coated surfaces. However, further microtribology tests are needed to confirm this assumption. Although scratch tests have been documented for the evaluation of dental gypsums and die hardeners, most of them were done with different tips under relatively heavy loads and some of the analyses were based on gross weight loss of the specimen ${ }^{7-9)}$. Heavy loads influence beyond the die hardener penetrated layer while weight loss analysis was less accurate. All of these limitations of the experimental design made previous results inconclusive. In contrast, the microscratch test under a load of 15 grams had a penetration depth of around $3 \mu \mathrm{m}$ (data from nanoindentation with a Berkovich tip under $150 \mathrm{mN}$ load), which is within the penetrated layer of the die hardener. This ensures the accuracy of the results as the reflection of the antiabrasive behaviour of the surface die hardener film and the die hardener penetrated layer only.

As illustrated in Fig. 4b (white arrow), the film was detached from the surface by abrasion, which indicated that the resin did not have strong physical or chemical bonding with the die stone surface. Therefore, it is not surprising that there was no improvement of the flexural strength after coating the specimens with the die hardener. Theoretically, the penetrated resin and the thin film on the surface would have the ability to seal surface defects. However, without the strong ability to bond the gypsum crystals, die hardener could not restrict the surface crack propagation under tensile stress. As a result, the die hardener did not improve the overall flexural strength of the specimens.

\section{CONCLUSION}

This is the first micro-mechanical investigation on the die hardener penetrated layer. From the current mechanical and SEM investigation, it can be concluded that the penetration of the die hardener into the surface of die stone did not improve mechanical properties of die stone. However, the die hardener film on the surface of die stone did have the ability to protect the surface of casts from abrasion damage.

\section{ACKNOWLEDGMENT}

Authors would like to thank the Otago Electron Microscopy unit and Ms. Liz Girvan for assistance using the SEM equipment. Constructive suggestions from two anonymous reviewers are also acknowledged.

\section{REFERENCES}

1) Prisco R, Cozzolino G, Vigolo P. Dimensional accuracy of an epoxy die material using different polymerization methods. J Prosthodont 2009; 18: 156-161.

2) Craig RG, Powers JM. Restorative Dental Materials, $11^{\text {th }}$ ed. St. Louis: Mosby Inc; 2002. p. 401-402.

3) Fukui H, Lacy AM, Jendresen MD. Effectiveness of hardening films on die stone. J Prosthet Dent 1980; 44: 5763.

4) Ghahremannezhad HH, Mohamed SE, Stewart GP, Weinberg R. Effects of cyanoacrylates on die stone. J Prosthet Dent 1983; 49: 639-646.

5) Sanad MEE, Combe EC, Grant AA. The effect of model sealant solutions on the properties of gypsum. J Dent 1980; 8: 152-157.

6) Harris PE, Hoyer S, Lindquist TJ, Stanford CM. Alterations of surface hardness with gypsum die hardeners. J Prosthet Dent 2004; 92: 35-38.

7) Lindquist TJ, Stanford CM, Knox E. Influence of surface hardener on gypsum abrasion resistance and water sorption. J Prosthet Dent 2003; 90: 441-446.

8) Lyon HE, Mitchell RJ, Patterson T. A comparison of abrasion resistance of dental stones. Dent Mater 1987; 3: 49-51.

9) Eames WB, Edwards CR, Buck WH. Scraping resistance of dental die materials: a comparison of brands. Oper Dent 1978; 3: 66-72.

10) Zinelis S, Eliades T, Eliades G, Makou M, Silikas N. Comparative assessment of the roughness, hardness, and wear resistance of aesthetic bracket materials. Dent Mater 2005; 21: 890-894.

11) Golovin Y. Nanoindentation and mechanical properties of solids in submicrovolumes, thin near-surface layers, and films: A Review. Phys Solid State 2008; 50: 2205-2236.

12) Bajada SB, Makinson OF. The effect of some surface treatments to dental modelling stones. Aust Dent J 1974; 19: 118-121. 